Effects of atomic interactions on the resonant tunnelling of sodium condensates

This content has been downloaded from IOPscience. Please scroll down to see the full text. 2005 J. Opt. B: Quantum Semiclass. Opt. 7 S59

(http://iopscience.iop.org/1464-4266/7/3/008)

View the table of contents for this issue, or go to the journal homepage for more

Download details:

IP Address: 192.167.18.242

This content was downloaded on 15/01/2016 at 10:07

Please note that terms and conditions apply. 


\title{
Effects of atomic interactions on the resonant tunnelling of sodium condensates
}

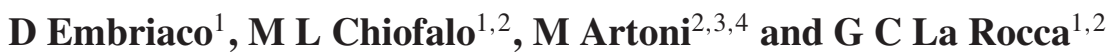 \\ ${ }^{1}$ Scuola Normale Superiore, Piazza dei Cavalieri 7, 56126 Pisa, Italy \\ ${ }^{2}$ Istituto Nazionale per la Fisica della Materia, Italy \\ ${ }^{3}$ Department of Physics and Chemistry of Materials, University of Brescia, 25133 Brescia, \\ Italy \\ ${ }^{4}$ European Laboratory for Non-Linear Spectroscopy, 50019 Sesto Fiorentino, Italy
}

Received 15 September 2004, accepted for publication 26 October 2004

Published 1 March 2005

Online at stacks.iop.org/JOptB/7/S59

\begin{abstract}
We investigate the influence of atomic interactions on the tunnelling of sodium condensates across a spatially oscillating optical barrier. In the limit of very fast barrier oscillations, in which resonant tunnelling via a metastable state takes place, the interactions affect the position and lineshape of the transmission peak. We anticipate that the possibility of modulating the interactions in a tunnelling condensate can be exploited to achieve nonlinear effects such as optical limiting and bistability.
\end{abstract}

Keywords: tunnelling, traversal time, matter waves, dynamic properties of Bose-Einstein condensates, atom lasers

\section{Introduction}

Resonant tunnelling occurs in a variety of different physical systems [1-3]. Resonant tunnelling across static double barriers, in particular, manifests as a peak in the transmission at energies which are resonant with the quasi-bound metastable states inside the double barrier and which typically fall well below the threshold for transparency of the single barriers. Such an effect has been extensively studied and exploited, e.g., to tailor the transport properties of low-dimensional semiconductor structures [4].

Resonant tunnelling is also possible when particles move across an external time-dependent potential. Potentials of the form $V(x, t)=U(x-l f(t))$, spatially oscillating according to a periodic function $f(t)$, may exhibit almost perfect transparency below the threshold that the barrier $U(x)$ would exhibit when at rest $[5,6]$. This generally occurs at very high frequencies of oscillation of the barrier where things work as if the particles were to tunnel across a frequency-independent double barrier described by a time-averaged potential.

For the specific case of a strong and rapidly oscillating square barrier resonant tunnelling of a (charged) particle has been predicted to occur in [7]. We have further examined the conditions for observing such a tunnelling effect using wavepackets of ultracold atoms impinging upon a spatially oscillating square light barrier [8], the relevant time-averaged potential being reported in figure 1. Recent advances in

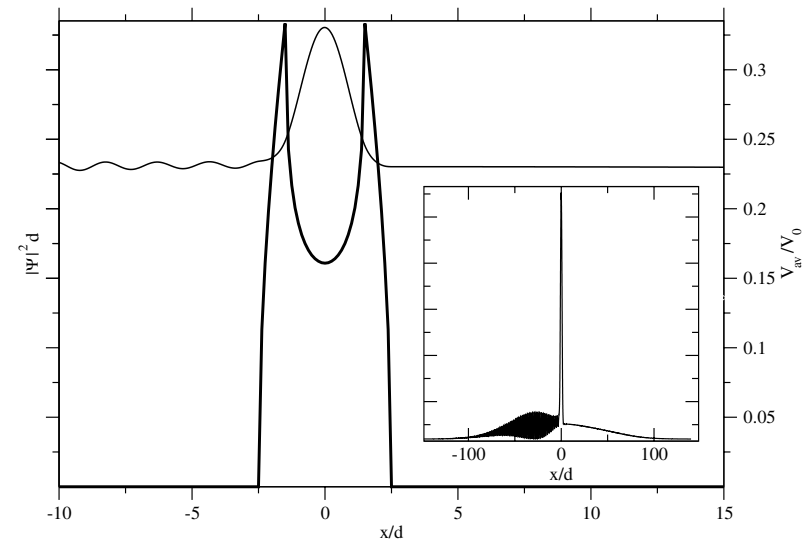

Figure 1. A snapshot of a tunnelling process, at the time in which the interacting atomic beam reaches a maximum in its density $|\Psi|^{2} d$ (light curve) inside the time-averaged potential $V_{\text {av }}$ (heavier curve). The zero of the atomic beam density is shifted in the plot at the energy $E=0.224 V_{0}>E_{0}$, corresponding to the position of the resonant tunnelling peak with $g / g_{0}=2$ in figure 4 . The inset shows $|\Psi|^{2} d$ on a larger scale.

manipulating the dynamics of atomic condensates with optical potentials $[9,10]$ could make tunnelling through time-varying optical barriers likely to be observed.

In this respect, tunnelling of condensates through moving light barriers provides an excellent starting point 
for uncovering novel tunnelling regimes not accessible in situations in which static barriers are used instead. The crossover region from slow to fast oscillating barriers, e.g., has recently been shown to exhibit so-far unexplored inelastic effects $[8,11]$. These, in turn, may possibly be exploited toward the efficient generation of atom laser sidebands in the intermediate frequency regime [11]. These can be related to the next term in the Floquet expansion of the periodic timedependent potential [12].

Resonant tunnelling of ultracold atoms is furthermore interesting due to the fact that atoms do interact with one another. The atom interaction strength, in addition, can easily be adjusted, which makes ultracold atoms quite suitable for studying tunnelling of many interacting particles. Under normal conditions, in fact, the atom mutual interaction strength depends on parameters that are accurately known such as the ratio $\hbar / m$, or on parameters that can be controlled experimentally such as the scattering length $a$, determined by the low-energy scattering properties of the gas, and the number of atoms $N$.

In the present contribution we investigate the influence of atomic interactions in the tunnelling of an ultracold atomic beam across a time-dependent barrier. We also briefly address the possibility of achieving atom-optical limiting and bistability with Bose-Einstein condensates tunnelling through a time-dependent barrier [13]. We restrict the present analysis to the high-frequency limit, in which the time-dependent barrier gives rise to resonant tunnelling via a single metastable state.

\section{The physical system}

We study the dynamics of an atomic wavepacket that moves along an atomic waveguide and impinges across a square barrier of thickness $d$ and height $V_{0}$, the position of which undergoes harmonic oscillations of elongation $l$ and frequency $v$. This is described by the time-dependent potential

$$
\begin{gathered}
V(x, t)=U[x-l \sin (2 \pi v t)] \\
U(x)=V_{0}[\theta(x-0.5 d)-\theta(x+0.5 d)],
\end{gathered}
$$

that in the $v \rightarrow \infty$ limit can be approximately mapped into the time-averaged potential $V_{\mathrm{av}}(x)=T^{-1} \int_{0}^{T} V(x, t) \mathrm{d} t$, with $T^{-1}=v$. Using a square barrier does not introduce appreciable differences with respect to the case of a more realistic Gaussian shaped optical barrier [8]. Yet, appropriate tailoring of both barrier dynamics and the atomic beam are needed to observe clearly the field-induced transparency. First, one should have $l>d$ to attain a double-barrier structure for the averaged form $V_{\mathrm{av}}(x)$ of the potential. Then $V_{0}$ and $l$ should be chosen so as to create only one quasi-bound (metastable) state of energy $E_{0}$ and width $\Gamma_{0}$ which lies below the maximum value of $V_{\mathrm{av}}(x)$. In addition, the energy spread $\Delta E$ of the incident wavepackets, such as those obtained from a Bose-Einstein condensate (BEC) through a continuous output coupler [15], must be sufficiently smaller than the metastable state linewidth. A typical set of parameters that satisfies the above requirements for noninteracting atomic samples was given in [8] for sodium and it is reported for convenience in table 1 . The values of the energy $E_{0}$ and linewidth $\Gamma_{0}$
Table 1. Barrier $\left(d, V_{0}\right)$ and resonance tunnelling $\left(E_{0}, \Gamma_{0}\right)$ parameters for the double barrier of figure 1 while $a$ denotes the scattering length [14] for sodium atoms ( $m=23 \mathrm{au}$ ) [8].

\begin{tabular}{lllll}
\hline$d(\mathrm{~nm})$ & $V_{0} / h(\mathrm{kHz})$ & $E_{0} / h(\mathrm{kHz})$ & $\Gamma_{0} / h(\mathrm{kHz})$ & $a(\mathrm{~nm})$ \\
\hline 827 & 4.2 & 0.90 & 0.06 & 3.29 \\
\hline
\end{tabular}

of the metastable state in table 1 refer to the case in which atomic interactions are immaterial, i.e. for very low densities. We note that all the parameters in table 1 can be scaled to represent other atomic species. Besides the barrier oscillation frequency $v$, the relevant parameters determining the features of our results are $\hbar^{2} /\left(2 m d^{2} V_{0}\right) \simeq 0.077$, the ratios $l / d=2$ and, most important, the scattering length $a$ describing the atomic interactions.

It is the aim of the present paper to elucidate the effects of the interactions on the resonant tunnelling. In particular, we assume here repulsive atomic interactions. When a significant atomic density builds up within the barrier region due to the resonance (cf figure 1), the mean field interaction shifts the metastable level to energies above $E_{0}$. As shown in detail below, such a nonlinear effect is however also accompanied by a broadening of the resonance. In the following we consider a wavepacket formed by a BEC of quantum degenerate bosonic atoms. The dynamical behaviour of a dilute BEC is accurately described by the time-dependent Gross-Pitaevskii equation for the atomic wavefunction $\Phi(\mathbf{r}, t)$ [9]. The use of an atomic waveguide and the interacting nature of the $\mathrm{BEC}$ require some additional modelling. The atomic waveguide reduces the dimensionality of the problem from three dimensions to one (1D), provided that the typical confinement energy in the plane perpendicular to the propagation direction $\hat{x}$ is much larger than the average interaction energy. Under this condition, no transverse modes can be excited during the tunnelling process, and we may then decompose $\Phi(\mathbf{r}, t)=\phi(y, z) \Psi(x, t)$ into a frozen transverse part $\phi(y, z)$ and a 1D time-dependent part $\Psi(x, t)$ [16]. Thus the 1D Gross-Pitaevskii equation for $\Psi(x, t)$ reduces to

$\mathrm{i} \hbar \frac{\partial}{\partial t} \Psi(x, t)=\left[-\frac{\hbar^{2} \nabla^{2}}{2 m}+V_{\mathrm{e}}(x, t)+g|\Psi(x, t)|^{2}\right] \Psi(x, t)$.

In equation (2), the external potential $V_{\mathrm{e}}(x, t)$ may be either the moving potential barrier $V(x, t)$ given in (1) or the timeaveraged potential $V_{\mathrm{av}}(x)$.

The nonlinear term $g|\Psi(x, t)|^{2}$ accounts for the atomic interaction whose strength is determined by the value of $g$. An immediate effect of the interactions on a trapped BEC at equilibrium is to shift the position of the discrete levels of the confining potential from their bare value in the absence of the atomic sample. For a 3D sample of $N$ atoms of mass $m, g^{3 \mathrm{D}}=4 \pi \hbar^{2} a \mathrm{~N} \mathrm{~m}^{-1}$ is completely determined by the system parameters with $a$ the scattering length. A reasonable estimate of its $1 \mathrm{D}$ reduction can be obtained by inserting the ansatz $\Phi(\mathbf{r}, t)=\phi(y, z) \Psi(x, t)$ in the 3D equation. The nonlinear term in the resulting equation for $\Psi(x, t)$ has the form $g^{3 \mathrm{D}}\left(\int|\phi(y, z)|^{4} \mathrm{~d} y \mathrm{~d} z\right)|\Psi(x, t)|^{2}$ [16]. Requiring that the $1 \mathrm{D}$ model system and the original $3 \mathrm{D}$ beam are 


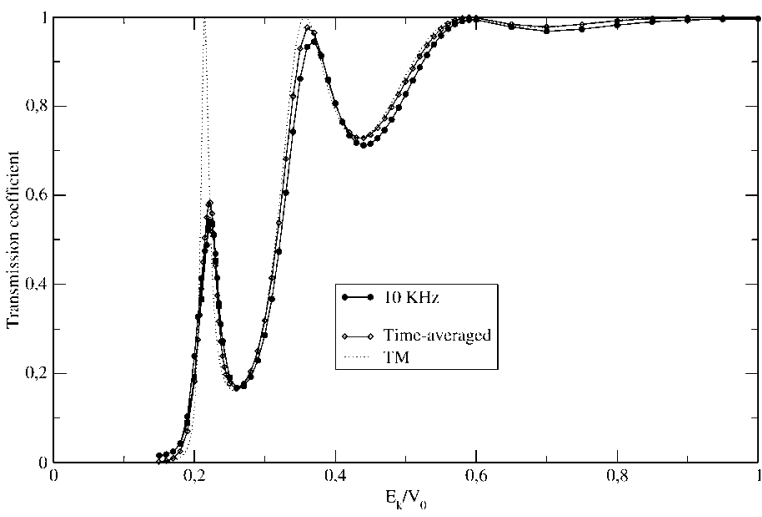

Figure 2. Resonant tunnelling of a Gaussian beam of Bose-Einstein condensed, interacting sodium atoms with $\Delta E=2 \Delta E^{*}$ $\left(\Delta E^{*} \simeq 0.0039 V_{0}\right)$ and $g / g_{0}=1$ (see the text). The simulated transmission coefficient is displayed for the beam tunnelling across the oscillating barrier at high frequency (circles) and across the corresponding time-averaged potential (diamonds). The transfer matrix (TM) results for a monochromatic noninteracting beam are also shown (dotted curve).

characterized by the same value of the average interaction energy $E_{I}^{3 \mathrm{D}}=E_{I}^{1 \mathrm{D}}=E_{I}$, with

$$
E_{I}^{1 \mathrm{D}}=g \frac{\int|\Psi(x)|^{4} \mathrm{~d} x}{\int|\Psi(x)|^{2} \mathrm{~d} x}
$$

and

$$
E_{I}^{3 \mathrm{D}}=g^{3 \mathrm{D}} \frac{\int|\Phi(\mathbf{r})|^{4} \mathrm{~d} \mathbf{r}}{\int|\Phi(\mathbf{r})|^{2} \mathrm{~d} \mathbf{r}}
$$

we are led in 1D to the relation $g=g^{3 \mathrm{D}}\left(\int|\phi(y, z)|^{4} \mathrm{~d} y \mathrm{~d} z\right)$. For a normalized anisotropic Gaussian wavepacket with transverse oscillator length $a_{\perp}$, we have $\int|\phi(y, z)|^{4} \mathrm{~d} y \mathrm{~d} z=$ $\left(\pi a_{\perp}^{2}\right)^{-1}$ and thus

$$
g=\frac{g^{3 \mathrm{D}}}{\pi a_{\perp}^{2}} .
$$

Reasonable values for $a_{\perp}$ validating the use of the $1 \mathrm{D}$ equation (2) can be obtained from the condition that the typical confinement energy $E_{c} \equiv \hbar^{2} /\left(m a_{\perp}^{2}\right)$ be much larger than

$$
E_{I}=\frac{4 \pi \hbar^{2} a N}{m} \frac{1}{\sqrt{2 \pi^{3}} a_{\perp}^{2} a_{h 0}} .
$$

Here, the longitudinal spread $a_{h 0}$ of the wavepacket is in turn related to its energy spread $\Delta E$ and initial wavevector $k_{0}$ through the expression $a_{h 0}=\hbar^{2} k_{0} /(2 m \Delta E)$. Using the value $\Delta E=\Delta E^{*} \simeq 0.016 \mathrm{kHz} \simeq 0.0039 V_{0}$ in equation (6) and a $1 \mathrm{D}$ interaction parameter $g \simeq 2 g_{0}$ in our simulations, where $g_{0} \equiv \hbar^{2} /(2 m d)$, we find that the above condition is satisfied by $a_{\perp} \simeq 1 \mu \mathrm{m}$ and a weak outcoupled beam with $N \simeq 100$ sodium atoms. Alternatively, one may tune $a$ within positive values by means of a Feshbach resonance mechanism [17], allowing a scaling of $N$ to different values.

We now proceed to discuss the tunnelling behaviour of the atomic beam resulting within the model system described above, after solving equation (2) for $\Psi(x, t)$ by means of a highly performing, explicit time-marching algorithm [18]. This algorithm is able to account for wavepacket deformations during the fast nonlinear dynamics underlying these tunnelling processes.

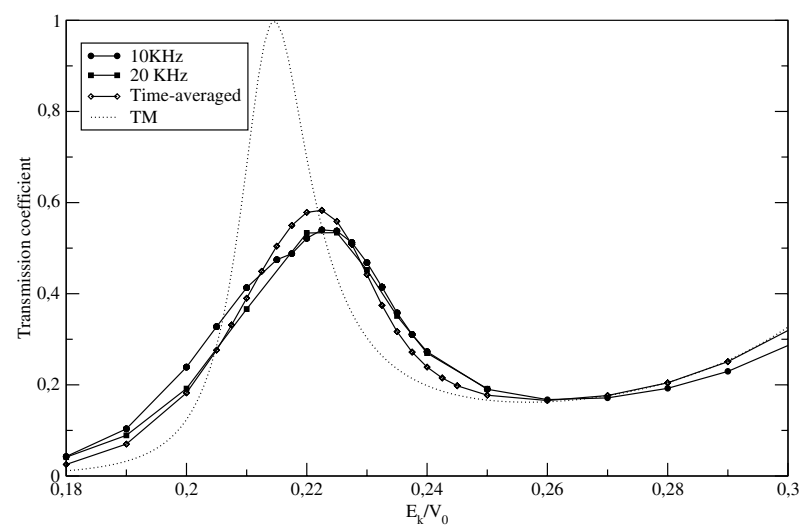

Figure 3. An enlarged view of figure 2 in the region around the resonant peak.

\section{The high-frequency limit in the presence of interactions}

We first test the validity of the time-averaged potential for describing the high-frequency limit in the presence of interactions. To this end, we prepare Gaussian wavepackets of sodium atoms with central wavevector $k_{0}$ and energy width $\Delta E$, where $k_{0}$ is varied so as to make the energy $E_{\mathrm{k}}=\hbar^{2} k_{0}^{2} / 2 m$ span the whole range $V_{0}$, the height of the barrier at rest. We thus simulate the tunnelling dynamics across the double barrier $V_{\mathrm{av}}(x)$ resulting from the time-averaged approximation, and repeat the simulation across the barrier oscillating at frequencies $v$ much higher than the inverse tunnelling time through the barrier when at rest. As this is estimated to be of the order of a few milliseconds, we may anticipate that the high-frequency regime takes place for $v>10 \mathrm{kHz}$ [8].

The transmission (coefficient) over the whole relevant range of energies is displayed in figure 2 as a function of $E_{\mathrm{k}} / V_{0}$ for a beam with spread $\Delta E=2 \Delta E^{*}$ and interaction coefficient $g / g_{0}=1$. We immediately notice in all cases the presence of a transmission peak around $E_{\mathrm{k}} / V_{0} \approx 0.2 \approx E_{0} / V_{0}$ (see table 1 ); this occurs well below the threshold for tunnelling across the barrier at rest and corresponds to a resonant tunnelling process. It can be demonstrated [8] that the second peak in the transmission is instead a precursor of tunnelling above threshold. The transmission curve obtained for the tunnelling across the time-averaged potential (diamonds) reproduces well the curve corresponding to tunnelling across the barrier oscillating at high frequency $v=10 \mathrm{kHz}$ (circles).

A blow-up around the first resonant peak is shown in figure 3. Slight differences between the time-averaged and the time-dependent cases appear even when doubling the oscillation frequency from 10 to $20 \mathrm{kHz}$, which did not occur instead in the case of a noninteracting atomic beam [8]. The nonlinearity in equation (2) due to the interactions makes the exchange of energy between the atomic beam and the oscillating potential barrier possible, thereby activating inelastic processes. This also explains why for the timedependent case the quality of the resonant peak is slightly worse, the peak looking lower and wider, than for the timeaveraged one. Similar conclusions obtain for an atomic beam with larger interaction coefficient $g / g_{0}=2$. The above 


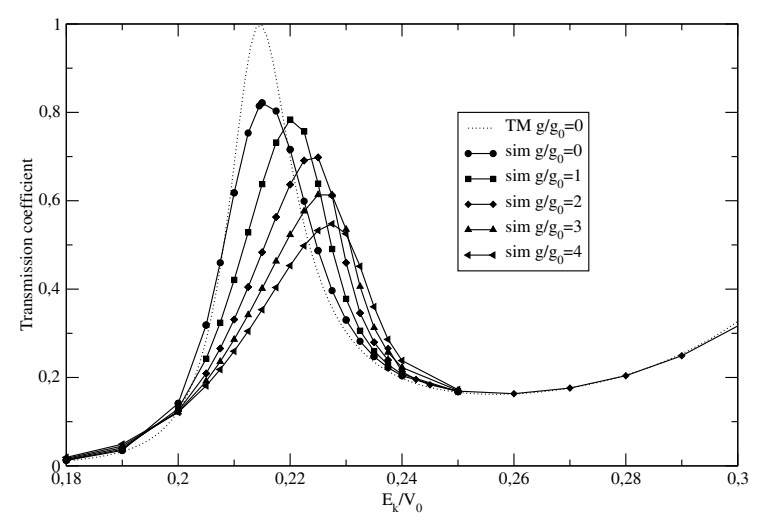

Figure 4. Resonant tunnelling of a Gaussian beam of Bose-Einstein condensed, interacting sodium atoms with $\Delta E^{*} \simeq 0.0039 V_{0}$ crossing the time-averaged potential (high-frequency limit). An enlarged view of the transmission coefficient in the region of the resonant tunnelling peak is shown for increasing values of the interaction parameter up to $g / g_{0}=4$. The transmission curve for the monochromatic noninteracting beam is also displayed by the dashed curve.

discussion indicates that even for an interacting beam, the physics of the tunnelling across $V_{\mathrm{av}}$ reproduces the main features of the time-dependent process, and is thus a good approximation in the high-frequency regime.

The main result emerging from figures 2 and 3 is however a shift of the peak position with respect to the case of a noninteracting monochromatic beam. The latter is displayed by a dotted curve and obtained through standard transfer matrix methods. The shift is particularly evident in figure 4. This yields an enlarged view of the transmission curve around resonance for atomic beams tunnelling through the timeaveraged potential and values of $g$ increasing up to $g / g_{0}=4$. The transmitted peak position is seen to shift towards higher energies and this can be understood by noticing that the energy of the metastable level, when the atomic wavepacket is inside the double barrier, experiences a mean field blue shift in the presence of the atom repulsive interactions. In order to match the blue-shifted resonant energy level ${ }^{5}$ the energy of the incoming atoms should then increase with respect to the value $E_{0}$ associated with the noninteracting beam (table 1). The enhancement of the atomic beam density while the atoms cross the resonance region is clearly evident from figure 1 leading to an appreciable $E_{I}$ (see equation (3)). The values of $E_{I}$ calculated from equation (3) corresponding to the snapshot in figure 1 are consistent with such an explanation.

The transmission lineshape flattens instead as $g$ increases. In fact, besides determining an enhancement of the total energy, repulsive interactions increase the energy width of the atomic wavepacket. Only the portion of atomic beam with energies compatible with the finite widths of the metastable state can be transmitted. The role of the energy width of the wavepacket is clearer in figure 5. This shows a blow-up of the transmission coefficient in the region of the resonant tunnelling peak for a beam with fixed interaction coefficient $g / g_{0}=2$, and two different values of $\Delta E=\Delta E^{*} \simeq 0.0039 V_{0}$ (dashed curve) and $\Delta E=2 \Delta E^{*}$ (solid curve). The peak with larger $\Delta E$

\footnotetext{
5 We have verified that the difference between the kinetic energy $E_{\mathrm{k}}$ and the
} total energy $E$ for the incident beam is negligible.

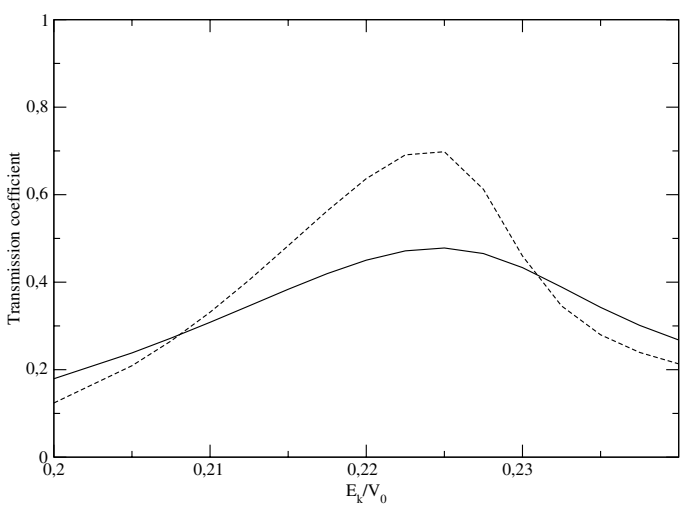

Figure 5. Resonant tunnelling of a Gaussian beam of Bose-Einstein condensed, interacting sodium atoms with $g / g_{0}=2$ fixed and two different values of the energy widths of the atomic beam, $\Delta E=\Delta E^{*} \simeq 0.0039 V_{0}$ (dashed curve) and $\Delta E=2 \Delta E^{*}($ solid curve).

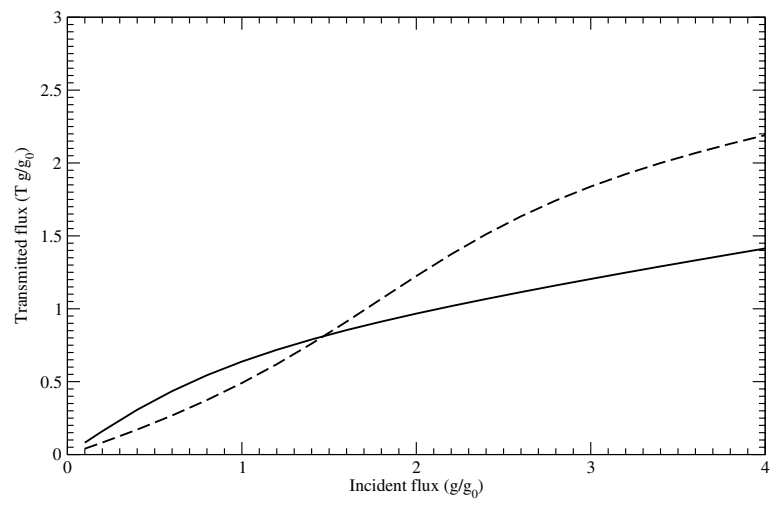

Figure 6. The output flux, measured as $T\left(E_{\mathrm{i}}\right) g / g_{0}$ from the transmission function $T$ at the energy $E_{\mathrm{i}}=E_{0}$ of the resonant peak in the empty double barrier, is displayed versus the incident flux $g / g_{0} \propto N$ (solid curve), showing saturation. The dashed curve shows the same at $E_{\mathrm{i}}=0.2275 V_{0}>E_{0}$ (see the text).

not only flattens, but also experiences a slight shift in position. In fact, a beam with a larger $\Delta E$ corresponds to a spatially narrower wavepacket, and thus a larger value of the peak density. With $g$ fixed in equation (3), this also yields increasing values of the interaction-driven mean field shift.

In the present high-frequency regime, where the resonant effect is expected to be clearly visible, a tuning of the interactions can be exploited to achieve atom-optical limiting and bistability [13] with time-dependent barriers. Optical limiting occurs when the output signal tends to saturate with increasing incident flux. If the incident beam energy $E_{\mathrm{i}}$ is close to the resonant value $E_{0}$ corresponding to the empty double barrier, an increase in the incident flux will have a negative feedback on the output signal, tending to depress it. This limiting effect for our interacting atomic beam is visible in figure 6 (solid curve), where we plot the incident flux measured as $g / g_{0} \propto N$, and the output flux $T\left(E_{\mathrm{i}}\right) g / g_{0}$ determined from the transmission curves $T$ at fixed energy $E_{\mathrm{i}}=E_{0}$ while varying $g$ in figure 4 . In the opposite case of a larger incident energy $E_{\mathrm{i}}>E_{0}$ corresponding to the peak position of the $g / g_{0}=1$ curve, the transmission is actually initially enhanced with increasing incident flux before starting to be suppressed (dashed line in figure 6). Such a behaviour 
Effects of atomic interactions on the resonant tunnelling of sodium condensates

is a precursor of optical bistability which, due to the widening and flattening of the resonant peaks in figure 4, does not fully develop.

\section{Concluding remarks}

The problem of a particle interacting with a time-modulated barrier has received much attention in recent years. In particular, we examine here the conditions under which a Bose-Einstein condensed sample of interacting atoms may resonantly tunnel across an oscillating light barrier. This extends earlier work of ours [8] in which atomic collisions were neglected, making the present investigation of the resonant tunnelling effect a more realistic one. As in the noninteracting case [8], tunnelling across the barrier occurs at energies well below the transparency threshold of the barrier at rest, yet atomic interactions appear to affect the transmission peak position and lineshape. We further exploit such a resonant tunnelling effect to anticipate that atom-optical limiting and bistability with Bose-Einstein condensates [13] could possibly be observed.

Owing to the recent progress in controlling the dynamics of condensates in optical potentials [10] and in manipulating the interaction properties of atomic beams by varying either the number of atoms or the scattering length through Feshbach resonances [17, 19], we believe in fact that the observation of such a resonant tunnelling effect is likely to occur. This would not only provide new insights into the dynamical properties of ultracold atoms moving across a barrier [9] but also lead to the observation of new phenomena such as atom laser sidebands [11], atom-optical limiting and bistability with condensates [13].

\section{Acknowledgments}

We would like to thank Professor F Bassani for useful discussions and comments.

We also acknowledge financial support from the EU (Contract HPRICT1999-00111), the INFM (Project PRA-
PHOTONMATTER) and MIUR (ACTIONE INTEGRADA ITALIA-SPAGNA IT 603).

\section{References}

[1] Chang L L, Esaki L and Tsu R 1992 Phys. Today 45 (10) 36

[2] Mizuta H and Tanoue T 1995 The Physics and Applications of Resonant Tunnelling Diodes (Cambridge: Cambridge University Press)

[3] Chang L L et al 1974 Appl. Phys. Lett. 24593

[4] See e.g. Yu P Y and Cardona M 1996 Fundamentals of Semiconductors: Physics and Materials Properties (Berlin: Springer)

[5] Pimpale A et al 1991 J. Phys. A: Math. Gen. 243533

[6] Ge J-Y et al 1996 J. Chem. Phys. 1058628

[7] Vorobeichik I et al 1998 Europhys. Lett. 41111

[8] Chiofalo M L et al 2003 New J. Phys. 578

[9] There is a wide literature on this subject. Review references could be Martellucci S et al (ed) 2000 Bose-Einstein Condensates and Atom Lasers (Dordrecht: Kluwer) Raizen M G 2000 Phil. Mag. B 802109

[10] A non-exhaustive selection of references on the manipulation of ultracold atomic beams is Anderson B P and Kasevich M A 1998 Science 2811686

Davies H J, Szymaniec K and Adams C S 2000 Phys. Rev. A 6213412

Greiner M, Mandel O, Esslinger T, Hänsch T W and Bloch I 2002 Nature 41539

Burger S et al 2001 Phys. Rev. Lett. 864447

Cataliotti F S et al 2001 Science 293843

Ferlaino F et al 2002 Phys. Rev. A $6611604 \mathrm{R}$

Fort C et al 2003 Phys. Rev. Lett. 90140405

[11] Chiofalo M et al 2004 J. Mod. Opt. 511083

[12] Büttiker M and Landauer R 1982 Phys. Rev. Lett. 491739

[13] Carusotto I et al 2000 Phys. Rev. Lett. 84399

[14] Pitaevskii L and Stringari S 2003 Bose-Einstein Condensation (Oxford: Clarendon)

[15] Bloch I et al 1999 Phys. Rev. Lett. 823008

[16] Jackson A D et al 1998 Phys. Rev. A 582417

[17] Feshbach H 1958 Ann. Phys. 5357

Feshbach H 1962 Ann. Phys. 19287

Feshbach H 1992 Theoretical Nuclear Physics (New York: Wiley)

[18] Cerimele M M et al 2000 Phys. Rev. E 621382

[19] Roberts J L et al 2001 Phys. Rev. Lett. 864211 Donley E A et al 2002 Nature 417529 